\title{
Concurrent Negotiation using Software Agents in Cloud
}

\author{
A. G. Patane \\ MBES college of Engineering, \\ Ambajogai \\ Maharastra
}

\author{
B. M. Patil \\ MBES college of Engineering, \\ Ambajogai \\ Maharastra
}

\begin{abstract}
In today's cloud environment, multiple service providers are available; among these most favorable service should be allocated to consumers as per their requirement. By introducing software agents, consumer and the provider are negotiating with each other to meet the requirement. The exactly favorable market discovered using cloud ontology, which gives the similarities between consumer services and provider services. Based on similarity reasoning, compatibility reasoning and numerical reasoning multiple cloud services are extracted by agents. Agents can negotiate with providers by focused selection of contract based on service capability tables (SCT) of consumer, provider and broker agents. Agents can also supports parallel negotiation between consumer-broker and multiple broker-producers. Concurrent negotiation helps to understand the bargaining position of consumer and regression based coordination will be done at broker side by considering minimum amount of penalty. The result shows that RBC at broker side gives higher performance in terms of utility and approves proposal with minimum penalty fee. It gives best results as compare to traditional utility oriented coordination.
\end{abstract}

\section{Keywords}

Cloud computing, Software agents, Concurrent negotiation Regression coordination.

\section{INTRODUCTION}

Basically cloud is collection of interconnected computers in network and cloud computing is collection of interconnected and virtualized computers which provides resource dynamically as per user requirements. This allocation of resources is done with some decisions based on similarities it may be based on numerical or compatibility. Negotiation means the communication between agents to meet some requirements. The negotiation between the participants is done using service level agreement [1]. As we know that cloud computing has different types in which cloud is being utilizes in the form of

- Software as a service - it delivers software which are remotely accessed via internet

- Platform as a service - it provides programming level platforms or API s to developers

- Infrastructure as a service - it provides the computational resources

- Communication as a service - it provides network bandwidth, network security, encryption and network management.

- Data as a service - It allows data to be stored at remote location.

In agent based cloud computing all resources from the provider side are made available to the consumer's through software agents. Each and every participant have their own agents it means providers have provider agent consumers have consumer agent and the communication between provider and consumer is made easy using broker agents in between them. Agents are responsible for the service discovery and the negotiation between provider and consumers. Using agents cloud service utilization is done in proper manner.

The cloud service life cycle has service requirements, service discovery, service negotiation, service composition, and service consumption. In service requirement phase consumer gives their requirement then those requirements passed to service discovery phase. Using service negotiation and service composition actual services are consumed by consumers. Service discovery is done with similarity, compatibility, functionality, numerical reasoning. After these matching finally the cloud resource is available to the consumers. Service discovery is done also using cloud ontology. Cloud ontology is the hierarchy of interrelated cloud service, which shows similarities between cloud services in term of hierarchy. Similarity, numerical, compatibility reasoning, all are determined using cloud ontology. Service discovery phase leads to the negotiation phase in that phase concurrent negotiation between provider, broker and consumer agents is there using service level agreement (SLA). SLA's are the acceptable terms for satisfying service requirements [2].

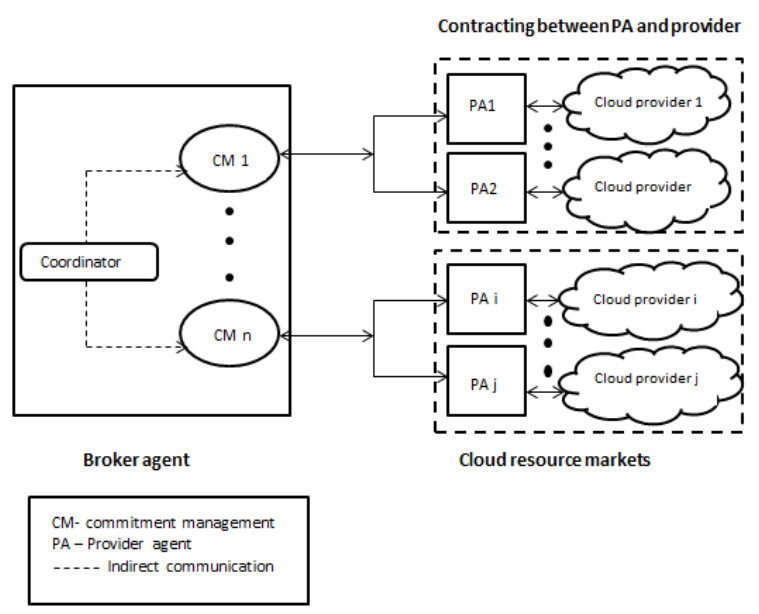

Fig 2: Concurrent negotiation of SLA's [1] [2].

This agents have their own capability tables with related information means consumer agent capability table contains information about brokers. Broker agent capability table contains all information about provider and resource information which are made available by the providers. Provider agent's capability table contains list of resources with their status either it may be waiting or busy.

Service composition phase deals with that the resources made available based on focused selection contract net protocol 
(FSCNP) [6]. Service capability table plays important role for focused selection of cloud resources.

\section{RELATED WORK}

The concept of searching or finding cloud resources is called cloudle and these cloud services extracted on basis of cloud ontology. The different reasoning types used to find out cloud resources called similarity reasoning, compatibility reasoning, numerical reasoning such cloudle with agents is developed in [1]. With the help of cloud ontology the cloud resources gets discovered and resources pooled towards users. It is formal representation of services that fulfills the requirements [2]. The cloud ontology consists of special definitions of the specific cloud concepts which are used to give the relationship among other cloud resources. It is also a self-discovery of resources based on cloud concept to meet the goal of cloud computing as SAAS that is software as a service [5].

An agent based cloud computing uses agents that is representative of each participant, for the communication between all the participants in cloud computing for strengthening discovery of cloud resources and service level agreement (SLA) negotiation. Sim proposed the agent based approach which is used mainly for strengthening cloud service discovery, service negotiation, and service composition. Service discovery is done on the basis of ontology concept and service reasoning i.e. similarity reasoning, compatibility reasoning and numerical reasoning, different agents used for service negotiation, all communication between different participant is done using agents and the negotiation protocol [6]. Similarly the grid resource allocation uses service negotiation and acquisition protocol (SNAP) which searches resource requirements of consumers of grid with proper utilization of those resources. The SNAP uses the SLA for operating resources [7]. For such specific searching of resources is done with contract net protocol also i.e. CNP it solves problem of communication and allocates resources to meet the requirements. It plays only two roles: client and server. In that client which requests and manages the request to the server and server decides whether to give the contract or not to that particular client [8]. Also in CNP, agent broadcast service requesting message to all other agents then agent matching requirement gives reply to client.

The agents make the system easier to communicate each other and proper service allocation due to which overall performance gets improved. Agent based computing involves new concepts called SCT's i.e. service capability tables which contains different degree of knowledge of neighboring participants and knowledge about cloud services. J. Octavio Gutierrez-Garcia and Sim [9] gives the agent based cloud workflow using petri-net strategy. In this strategy concurrent and parallel management of cloud workflow using agent is introduced using traditional CNP. This strategy introduces tokens for transferring the messages between agents. Semi recursive contract net protocol SRCNP combines the features of SCT and CNP, and agents plays multiple roles and requests are submitted to specific number of agents. Also Sim proposed focused selection contract net protocol (FSCNP) devised for specifying the interactions of cloud agents. Also a FSCNP agent focuses services by interacting other agents by observing SCT's which provides relevant services, plays multiple roles and it restarts the contract if it gets failed at once. Unlike CNP, FSCNP sends requesting message to selected agents instead of broadcasting. This system designed for supporting negotiation between only two participants that are buyer and seller.
The complex negotiation mechanism has concepts called bargaining position estimation (BPE) which communicate in between consumer and broker agent and regression based coordination (RBC) for communication in between broker and provider agents in multiple resource markets. RBC strategy gives higher success rate than others. BPE allows many-tomany negotiation between consumer agents and broker agents. Concurrent negotiation is parallel negotiation between consumer, broker, provider agents and contracting the particular proposals within consumer's deadline. In BPE strategy agents responds with different market conditions and making proposal in adjustable amounts so that the penalty to brokers gets reduced.

\section{CONCURRENT NEGOTIATION USING SOFTWARE AGENTS}

There are three types of participants in agent based cloud computing and the negotiation between these three is using the negotiation protocol. Agent is some software agents which can communicate by passing messages to other participants. The three participants are named consumer agent, broker agent, and provider agent. Broker agent is third party who is responsible for the service composition phase in resource allocation.

Different phases of allocation of proper resources are to discover services, negotiation between agent and service composition. Service discovery phase the resources are discovered from multiple related e- markets which are most probable on the basis of reasoning such as similarity, compatibility, numerical reasoning and service ontologies as discussed in section 2. After discovery of services negotiation between particular focused groups of users is introduced. Instead of direct communication between service provider and consumers the broker agents are introduced. They can communicate on the basis of FSCNP means limited number of related agents only gets disturbed by the requesting message sent by consumer agent.

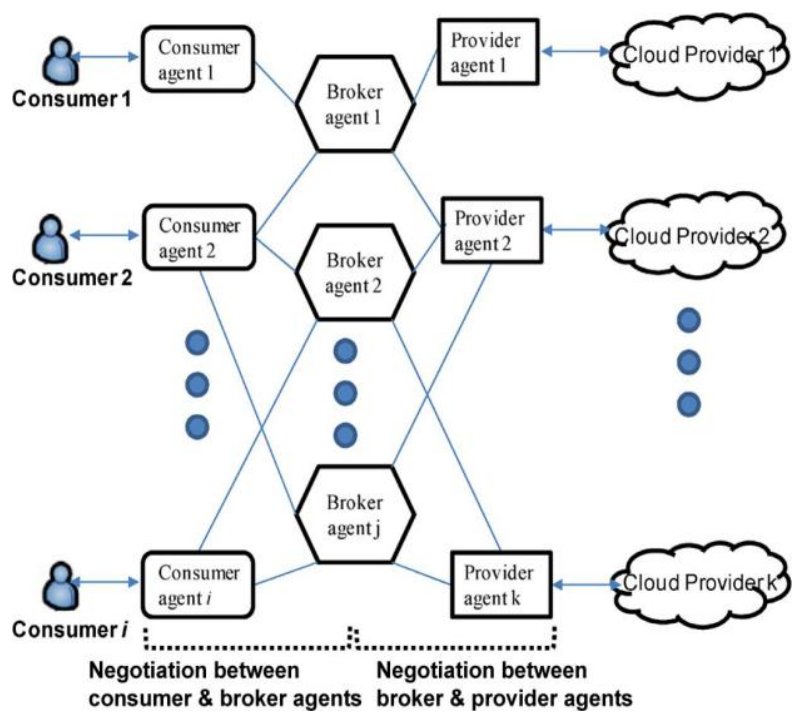

Fig 3: Agent based architecture [6] [3].

Basically agent based architecture consist of consumer agent, broker agent, provider agent as shown in Fig 3. Actual requesting participants are consumer agents, in between negotiating participants are the broker agents and providers of cloud services are provider agents which are representatives of cloud service provides. Many-to-many negotiation between 
agents is done using market oriented approach which regulates the supply and demand.

In [3], [10] simulation of cloud services with consumer requests is done using agent based cloud computing workflow. It has consumer agent for the purpose of requesting cloud services, broker agents accepts these requests and given to the focused number of cloud providers which meets requirements of consumer agents. Brokers purchases the cloud services and given it to consumers in reasonable costs with minimum loss to itself. Broker agents communicate in terms of cloud service market and cloud resource market [6]. In cloud service market broker negotiate with consumers with acceptable terms in SLA's for satisfying consumer request. And in cloud resource market broker communicate with resource provider for reserving resources.

Cloud negotiation is done within i) consumer agent and broker agent ii) Broker agent and provider agent. Steps to negotiation are:

- Negotiation completes in number of rounds.

- Initially proposals are introduced in alternate rounds at consumer and broker side.

- Multiple consumers can send their request to the multiple brokers simultaneously.

- Broker forwards that request to the probable possible deals which are most desirable.

- If any argument doesn't reached negotiation in next round is started. At any round concession is determined.

- Negotiation terminates when i) all requirements met ii) negotiation crosses the deadline without any requirement matching.

The negotiation between the participants is done by observing the SCT's at individuals.

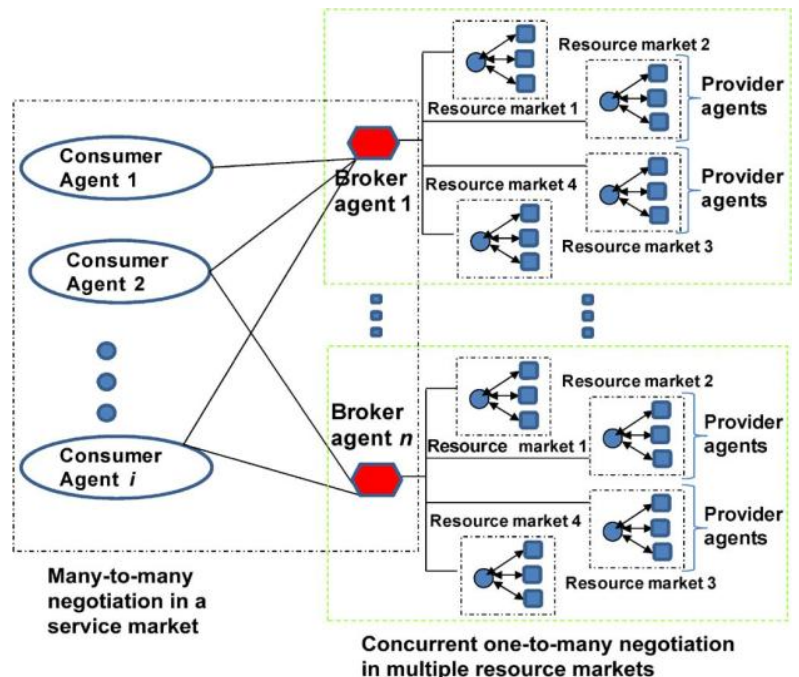

Fig 4: A cloud negotiation mechanism [6], [3].

1. Consumer agent's SCT: It consist of record list of broker agents but not service capability of broker agents so that all the brokers can subcontract the proposals.

2. Broker agent's SCT: It has SCT of provider agents with their information about location, service capability and status. Also it has SCT of other brokers with their locations.

3. Provider agent's SCT: It consist of SCT of other service provider and SCT of resources with their status either it may be busy or in waiting state.

4. Resource agent's SCT: It has the SCT of siblings under SPA's (service provider agent). Also has information about location, cost, and status.

These SCT's are used for focused selection of cloud services. The name focused selection contract net protocol because an agent consults with only limited number of relevant agents it does not broadcasts the requirement to all the agents. The consumer agent requests for call-for-proposals to broker agents then broker resubmit that proposal with some amount as a bid. This focused selection reduces the number of messages exchanged between the cloud agents and they communicate with only the relevant services. In FSCNP, an agent can play multiple roles such as accepting request post that requests to other agents or service providers by resubmitting the contract by applying bid cost to the same proposal. If the requirement gets matched to the service then that proposal is approved otherwise it is ready to next round with next bidding cost. The number rounds are calculated from initial round to final approval of that proposal with some penalty fee to the broker.

\section{COMMITMENT AND THE COORDINATION OF NEGOTIATION}

The commitment of the proposal is managed by algorithm 1 [11]:

At each negotiation round

- Determine the reneging probability of each provider agent.

- Expected utility or supporting required by the each provider agent proposal.

- If each proposal is acceptable then

i. Request is send to these provider agents by broker agent

ii. Wait until that proposal is confirmed.

- If broker receives confirmation then broker accepts that contract

Else broker resubmits contract with some concession.

For coordinating the proposals concurrent one-to-many negotiation is adopted. The coordination includes evaluating the change in expected penalty fee in each one-to-many negotiation and deciding whether to proceed or terminate the negotiation. Such coordination is achieved by using the regression based coordination [3]. It works as follows:

At each negotiation round and for each resource

- At each commitment it checks for whether the proposal is in acceptable range i.e. in initial price and reserved price.

- If proposal is acceptable for broker it will be at acceptable list of resources.

- If acceptable list is empty then coordination cannot complete the concurrent negotiation 
Else

i. Determine the change in parameters or utility.

ii. Decide whether to terminate or proceed.

Requested cost of proposal falls into initial price and reserved price then that proposal gets added to the allocated resource list of proposals. If proposal is accepted and if it is not present in resource table then it is considered that coordination does not completed into that negotiation. So that commitment with coordination is important together. The utilities required for next round and previous round are equal means utility oriented coordination and the regression based coordination has similar nature.

\section{EXPERIMENTAL ANALYSIS}

Initially consumers and providers communication is directly done without any third party called broker so that cost of requested resources is not negotiable. After introducing broker agents the cost is negotiable and they determine the utility. Utility is the most probable service with number of resources in reasonable cost. This utility function of RBC strategy is calculated in multiple negotiations round made available by using cost based on RBC. The best proposals received by the broker agent have minimum cost Min among provider agents of resource therefore the utility [6] is calculated by using:

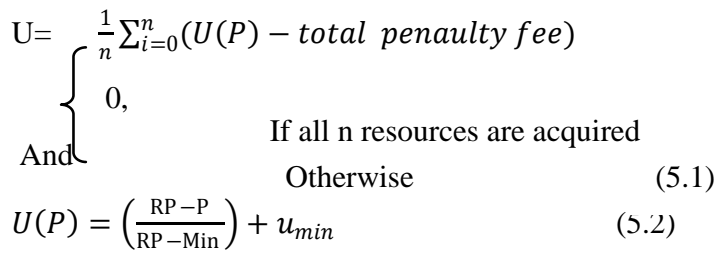

Where RP is reserve price at broker agent for resource $\mathrm{R}$, Min is minimum reserve price, $\mathrm{P}$ is cost of proposal at consumers and $u_{\min }$ is minimum requirements needed to approve the proposal that's why it should not be zero and it has minimum value of 0.1 . And the penalty fee is calculated with difference between cost at previous round and cost at current round. Then the total penalty fee at current round $t$ is calculated as:

Total penalty $f e e=\sum_{t=1}^{k}(P P-C P)$

Where $t$ is from first round to the final round $k$ at which resource at defined cost gets available, $\mathrm{PP}$ is bargaining price at consumer and the $\mathrm{CP}$ is current round's price at agent.

The experimental setup has values as shown in Table1. There are $n$ numbers of resources. For allocation of these resources there is reserved cost, minimum cost of proposals and minimum utilities which is not less than 0.1. Using these costs final utility with allocated no. of resources is determined.

Table1. Utilities number of resources

\begin{tabular}{|l|l|l|l|}
\hline Sr. No. & Variable & Description & values \\
\hline 1 & $n$ & No. of resources & {$[2,7]$} \\
\hline 2 & Min & $\begin{array}{l}\text { Minimum cost of } \\
\text { proposal }\end{array}$ & $\begin{array}{l}\text { Min }<\text { actual } \\
\text { cost }\end{array}$ \\
\hline 3 & $R P$ & $\begin{array}{l}\text { Reserve price of } \\
\text { proposal at broker } \\
\text { agent for resource }\end{array}$ & $\begin{array}{l}\text { RP>Min cost of } \\
\text { proposal }\end{array}$ \\
\hline 4 & $P$ & Cost of proposal & P>RP \\
\hline
\end{tabular}

\begin{tabular}{|l|l|l|l|}
\hline & & at consumer & \\
\hline 5 & $u_{\min }$ & $\begin{array}{l}\text { Minimum } \\
\text { requirements to } \\
\text { approve the } \\
\text { proposal }\end{array}$ & 0.1 \\
\hline 6 & $U(P)$ & $\begin{array}{l}\text { Final utility of } \\
\text { proposal }\end{array}$ & {$[0.6,0.66]$} \\
\hline
\end{tabular}

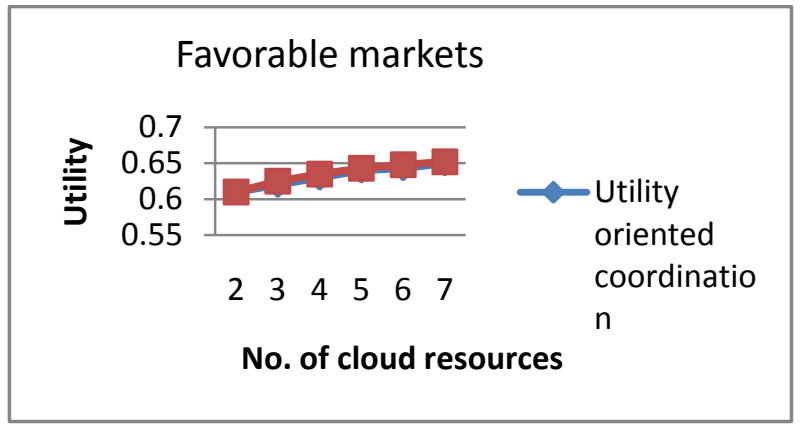

Fig 6: Performance graph in terms of utility in favorable markets.

From above graph it is observed that utility oriented coordination and the regression based coordination utilizes the resources having maximum utility in number of rounds, Provided that resource gets allocated to consumers within deadline provided by consumers. RBC achieves regression coordination between the broker and the provider and it allows giving the $\mathrm{RBC}$ cost which minimizes penalty fee to broker. Regression based coordination gives the resources matching with higher utilities as compare to simply utility oriented coordination.

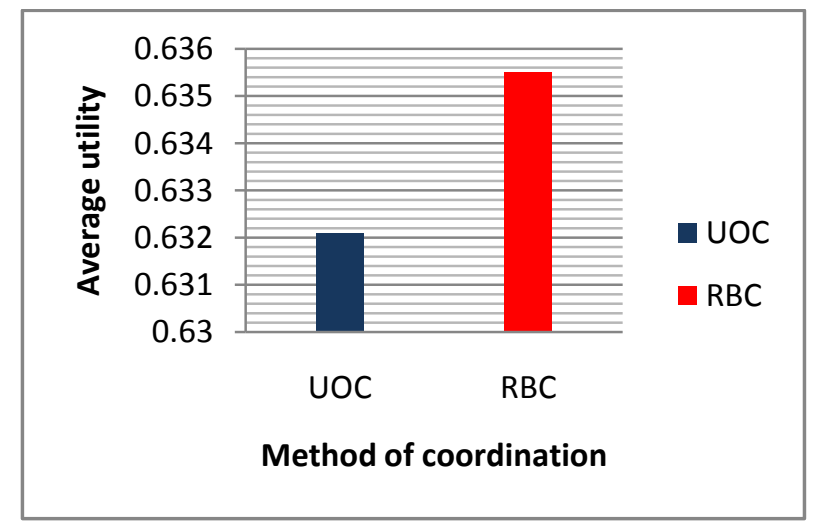

Fig 7. Comparison of two coordination methods in terms of average utility.

Avg.utility $=\frac{\sum_{i=2}^{n} U(P)}{\text { total no.of utilities }}$

For the calculation of average utility of coordination strategies (5.4) is used. In equation (5.4) $\mathrm{n}$ indicates no. of resources and $\mathrm{U}(\mathrm{P})$ is final utility of proposal. Above graph shows the comparison between average utility functions with two coordination methods that are UOC and RBC. Regression based coordination deals with maximum utilities that is benificial to the reduce the penalty fee to the broker agent because utility is the function of costs. And by using equation (5.3) the total penalty fee to the broker is calculated. 


\section{CONCLUSION}

By introducing agents in this system communication to cloud provider is possible at least one time even though resources are in busy state. The multiple cloud providers and multiple agents can fulfill the requirements of the consumers which pass the request to the focused or relevant users by observing SCT's so that other irrelevant not gets affected more. These multiple agents give the cooperative nature with regression based coordination and cloud services make available to consumers with reduced penalty fee to the brokers. Also if request does not completed in first round then there is facility to announce bargaining cost to the same so that request gets fulfilled in next subsequent rounds. So the BPE and RBC respond better to the all favorable resource market because they do not provide excessive concessions in markets. RBC gives the high performance in terms of utility and gets available to the consumers though broker agents with minimum penalty fee.

\section{REFERENCES}

[1] J. Kang and K.M. Sim, "Cloudle: An Agent-Based Cloud Search Engine that Consults a Cloud Ontology," Proc. Int'l Conf. Cloud Computing and Virtualization, pp. 312318, May 2010.

[2] K.M. Sim, "Towards Complex Negotiation for Cloud Economy," Proc. Int'l Conf. Advances in Grid and Pervasive Computing (GPC '10), R.S. Chang et al., eds., pp. 395-406, 2010.

[3] K.M. Sim, "Complex and Concurrent Negotiations for Multiple Interrelated E-Markets," IEEE Trans. Systems, Man and Cybernetics, Part B, preprint, 2012, doi:10.1109/TSMCB.2012.2204742.

[4] Octavio Gutierrez-Garcia and K.M. Sim " Agent -based cloud service compostion", Springer vol.22, no. 2, March/April 2012.
[5] L. Youseff, M. Butrico, and D. Da Silva, "Toward a Unified Ontology of Cloud Computing," Proc. Grid Computing Environments Workshop (GCE '08), pp. 110, 2008.

[6] Kwang Mong Sim “Agent based cloud computing” IEEE transactions on services computing, vol. 5, no. 4, october-december 2012.

[7] K. M. Sim " Relaxed-criteria G-negotiation for Grid Resource Co-allocation (Position Paper)" ACM SIGecom Exchanges, Vol. 6, No. 2, December 2006.

[8] R.G. Smith, "The Contract Net Protocol: High-Level Communication and Control in a Distributed Problem Solver," IEEE Trans. Computers, vol. C-29, no. 12, pp. 1104-1113, Dec. 1980.

[9] J.Octavio Gutierrez-Garcia and K.M. Sim "Agent-based cloud workflow execution", integrated Comuter-Aided Engineering 19 (2012) 39-36 DOI 10.3233/ICA-20120387.

[10] K.M. Sim and B. Shi, "Concurrent Negotiation and Coordination for Controlling Grid Resource CoAllocation," IEEE Trans. Systems, Man and Cybernetics, Part B, vol. 40, no. 2, pp. 753-766, June 2010.

[11] K. M. Sim, "Towards complex negotiation for cloud economy," in Proc.5th Int. Conf. Grid Pervasive Comput., vol. 6104, LCNS, pp. 395-406.

[12] J.O. Gutierrez-Garcia and K.M. Sim, "Agent-Based Service Composition in Cloud Computing," Proc. 2010 Conf. Grid and Distributed Computing, Dec. 2010.

[13] R. Buyya et al., "Cloud Computing and Emerging IT Platforms: Vision, Hype, and Reality for Delivering Computing as the $5^{\text {th }}$ Utility," Future Generation Computer Systems, vol. 25, no. 6, pp. 599- 616, June 2009. 